LA-UR- $01-3186$

c./ Title:

Author(s):

Submitted to:

MHD MODELING OF MAGNETIZED TARGET FUSION EXPERIMENTS

Peter T. Sheehey, Rickey J. Faeh1, Ronald C. Kirkpatrick, and Irvin R. Lindemuth

Proceeding of IEEE International Conference on Pulsed Power and Plasma Science "PPPS-2001"

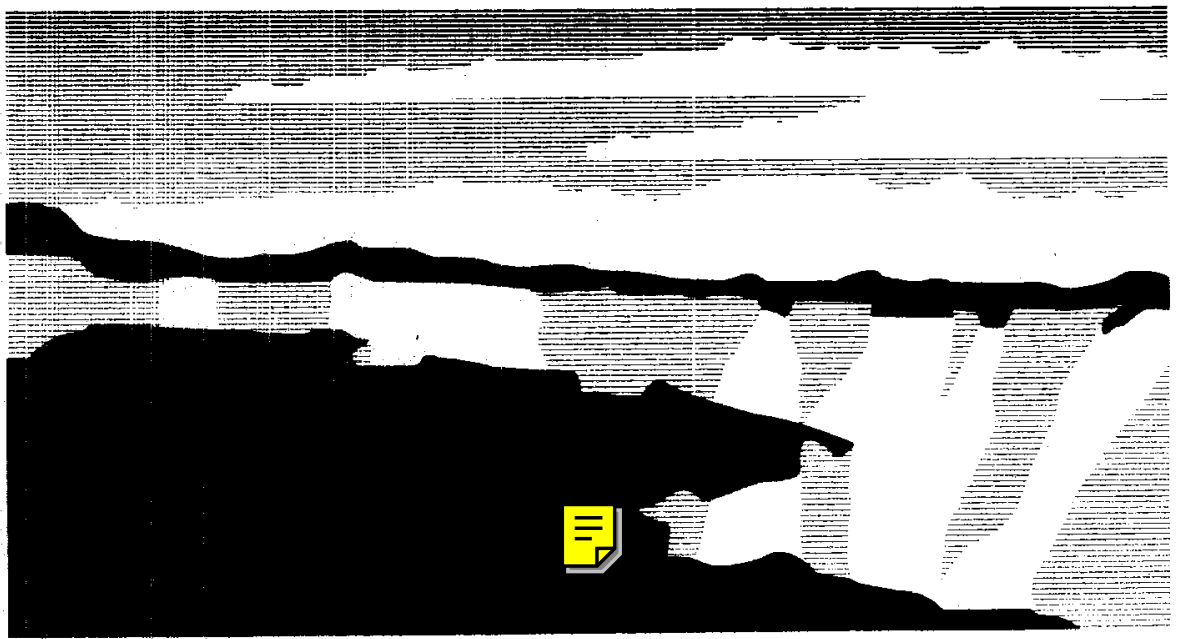

NATIONAL LABORATORY

Los Alamos National Laboratory, an aftirmative action/equal opportunity employer, is operated by the University of California for the U.S. Department of Energy under contract W-7405-ENG-36. By acceptance of this article, the publisher recognizes that the U.S. Government retains a nonexclusive, royalty-free license to publish or reproduce the published form of this contribution, or to allow others to do so, for U.S. Government purposes. The Los Alamos National Laboratory requests that the publisher identify this article as work pertormed under the auspices of the U.S. Department of Energy. 


\title{
MHD MODELING OF MAGNETIZED TARGET FUSION EXPERIMENTS
}

\author{
Peter T. Sheehey, Rickey J. Faehl, \\ Ronald C. Kirkpatrick, and Irvin R. Lindemuth \\ Los Alamos National Laboratory \\ Los Alamos, NM 87545 USA
}

\begin{abstract}
Magnetized Target Fusion (MTF) is an alternate approach to controlled fusion in which a dense $\left(O\left(10^{17-18}\right.\right.$ $\left.\mathrm{cm}^{-3}\right)$ ), preheated $(\mathrm{O}(200 \mathrm{eV}))$, and magnetized $(\mathrm{O}(100$ $\mathrm{kG})$ ) target plasma is hydrodynamically compressed by an imploding liner. If electron thermal conduction losses are magnetically suppressed, relatively slow $O(1$ $\mathrm{cm} /$ microsecond) "liner-on-plasma" compressions may be practical, using liners driven by inexpensive electrical pulsed power. Target plasmas need to remain relatively free of potentially cooling contaminants during formation and compression. Magnetohydrodynamic (MHD) calculations including detailed effects of radiation, heat conduction, and resistive field diffusion have been used to model separate target plasma (Russian MAGO, Field Reversed Configuration at Los Alamos National Laboratory) and liner implosion experiments (without plasma fill), such as recently performed at the Air Force Research Laboratory (Albuquerque). Using several different codes, proposed experiments in which such liners are used to compress such target plasmas are now being modeled in one and two dimensions. In this way, it is possible to begin to investigate important issues for the design of such proposed liner-on-plasma fusion experiments. The competing processes of implosion, heating, mixing, and cooling will determine the potential for such MTF experiments to achieve fusion conditions.
\end{abstract}

\section{INTRODUCTION}

Magnetized Target Fusion (MTF, also referred to by its Russian acronym MAGO, for magnetic compression) is an approach to controlled fusion that is intermediate between magnetic confinement and inertial confinement fusion (ICF) in time and density scales. Bigger targets and much lower initial target densities than in ICF can be used, reducing radiative energy losses. Reduced losses permit near-adiabatic compression of the fuel to ignition temperatures, even at low (e.g., $1 \mathrm{~cm} / \mu \mathrm{sec}$ ) implosion velocities. In MTF, the convergence ratio $\left(r_{\text {initial }} / r_{\text {final }}\right)$ of the pusher in quasi-spherical geometries may potentially be less than 10 , depending upon the initial temperature of the fuel and the adiabaticity of the implosion. Therefore, "liner-on-plasma" compressions, magnetically driven using relatively inexpensive electrical pulsed power, may be practical [1-4].

An MTF system requires two elements: (1) a preheated and magnetized initial "target" plasma; (2) a target implosion driver. Because the reduced energy losses in MTF relax the power and intensity requirements for an implosion driver, an optimal driver source for MTF might be relatively inexpensive electrical pulsed power, to drive a liner-on-plasma implosion. This could utilize either fixed pulsed-power facilities, such as Los Alamos' Atlas capacitor bank and the Shiva-Star capacitor bank at the Air Force Weapons Laboratory (Albuquerque), or explosive-flux-compression generators, such as the Russian 200-MJ-class disk flux compression generators $[3,4]$. Such energy-rich sources might allow a demonstration of fusion ignition via MTF, without a major capital investment in driver technology.

The success of magnetized target fusion will depend upon a number of issues. The initial target plasma must meet minimum temperature $(\sim 50 \mathrm{eV}$, preferably $100-300$ $\mathrm{eV})$, density $\left(\mathrm{O}\left(10^{17-18} \mathrm{~cm}^{-3}\right)\right.$, and magnetic field $(>50$ $\mathrm{kG})$ requirements, and must have a lifetime, adjacent to the supporting wall, greater than the implosion time (perhaps 5 to $50 \mu \mathrm{sec}$ for a pulsed-power-driven implosion, depending on the radius and speed of the imploding liner). Plasma-wall interaction must not cause excessive introduction and mixing of impurities, which might lead to rapid cooling of the plasma, either by bulk mixing with cool material or radiative cooling from high$\mathrm{Z}$ contaminants. As the liner implodes, it must remain sufficiently intact that it can effectively compress the target plasma.

Los Alamos National Laboratory (LANL), the Air Force Weapons Laboratory (Albuquerque), and the AllRussian Scientific Research Institute of Experimental Physics (VNIIEF) have pursued MAGO/MTF research in recent years [3-12]. The Field Reversed Configuration (FRC), an elongated compact toroid, is presently under development as an MTF target plasma at Los Alamos. The Air Force Weapons Laboratory (Albuquerque), collaborating with LANL, has been developing a liner driven by the Shiva-Star capacitor bank (and potentially in the future, by Atlas), which could compress this FRC or other suitable target plasmas. Work continues at VNIIEF on the Russianoriginated, explosive-pulsed-power-driven MAGO plasma formation scheme; experiment and theory are aimed at measuring and optimizing the suitability of the plasma created for MTF compression. Two other potential MTF target plasma schemes are not at present being actively investigated, although facilities exist on which this could be done: (1) a partially wall-supported deuterium-fiber-initiated Z-pinch [13,14]; (2) the beamdriven ICF capsule-like $\Phi$-target [15]. 
Related experimental and computational work aims to evaluate explosive-flux-compression generators and existing pulsed power facilities as MTF liner drivers. A joint LANL-VNIIEF experiment (high energy liner "HEL-1") executed in August, 1996, put approximately $100 \mathrm{MA}$ through a 24-cm-radius, 4-mm-thick, $10-\mathrm{cm}$ long aluminum cylindrical liner, which, by the time it reached the $5.5 \mathrm{~cm}$ radius of the central measuring (diagnostic) unit (CMU), was imploding at $7 \mathrm{~mm} / \mu \mathrm{sec}$ and contained approximately $20 \mathrm{MJ}$ of kinetic energy $[7,8,11,12]$. Such a liner approaches the energy and velocity regime required to drive an MTF target such as MAGO to fusion conditions. The recent liner experiments at Shiva-Star demonstrate liner performance (3 $\mathrm{mm} / \mu \mathrm{sec}$ ) suitable for longer-lived MTF target plasmas, such as the FRC.

In this paper, we describe computational modeling of proposed cylindrical liner-on-plasma compressions of such target plasmas with such liner systems. Computational models of the MAGO target plasma and the HEL-1 liner have shown good agreement with experiment [5-12]. These computational models provide good starting points for liner-on-plasma simulations. The results of such liner-on-plasma simulations can point out important issues that must be confronted in designing such experiments.

\section{COMPUTATIONS}

Several MHD codes have been employed in modeling MTF experiments. The Los Alamos MHRDR code has been used extensively in modeling the MAGO and fiber-initiated Z-pinch target plasma experiments [13]. Because MHRDR is fully implicit and has a generalized Eulerian structure, in which zonal quantities such as velocity are computed relative to a preprogrammable grid velocity, the code can run liner-onplasma calculations relatively quickly. However, it can only compute a single material (e.g., the target plasma DT); the outer radial boundary of the computational mesh is programmed to implode at the expected liner velocity. Boundary conditions are idealized: perfectly electrically conducting and zero-temperature, infinitely heat-conducting walls (hence the heat conductivity of the magnetized plasma is the only impediment to heat flow out the boundary). In these cases MHRDR uses cylindrical $r-z$ geometry with a single $B_{\theta}$ magnetic field component. The plasma radiative energy losses and electrical resistivity are taken from Los Alamos "Sesame" data tables. Heat conduction is full (arbitrary $\omega_{\alpha} \tau_{\alpha}$ ) Braginskii, unless we override this for comparison to other models.

A second Los Alamos Eulerian MHD code [16] was used to model the HEL-1 and Shiva-Star liner experiments. This code can compute multiple materials, such as an aluminum liner imploding onto a DT plasma. However, it runs much slower than MHRDR because it is not fully implicit and must use a fixed grid. This code has been used to do one- and two-dimensional liner-onplasma calculations of Atlas or HEL-1-like liners driving compression of MAGO-like target plasmas. At present,
Braginskii heat conduction is only included for the electron fluid (ion fluid has the non-magnetized conductivity value); however, it has been possible to estimate the Braginskii effect on the ions and include a constant factor times the non-magnetized value $(1 / 15)$ to approximate the full Braginskii magnetoinsulation effect. Diffusion of magnetic field, heat, and radiation between liner and plasma is computed

The MOQUI MHD code [17] has been used to model the formation and translation of the FRC being developed as a target plasma at Los Alamos. Although the initial FRC demonstration target plasma experiment at LANL will use a cylindrical theta-pinch coil, calculations are also being done with a conical thetapinch geometry, which facilitates translation of the FRC into the planned liner implosion area. In addition, the calculation shown in Figure 1 also has mirror symmetry about the center of the imploding liner area, so that two oppositely directed FRCs can collide there and for some values of resistivity (an adjustable model in the code), merge. These authors have not yet calculated liner-onplasma compressions of this FRC, although M. Friese using the MACH2 code has begun such calculations. Using the second Los Alamos MHD code described above, calculations were performed of the Shiva-Star liner implosion on an initial $B_{z}$ field of 5 Tesla. The resulting flux compression would cause return currents on the inner surface of the imploding liner greater than one $\mathrm{MA} / \mathrm{cm}^{2}$, which would cause melting/vaporization of the liner surface. Whether or not this would lead to unacceptable contamination of the FRC for MTF purposes will be answered by experiment and further detailed liner-on-plasma calculations.

One- and two-dimensional liner-on-plasma problems based on other demonstrated target plasma and liner drive quantities have been run with both Los Alamos MHD codes. Target plasmas resemble late-time (smaller chamber) computed MAGO plasmas: $O(100$ $\mathrm{eV}), 10^{-5} \mathrm{~g} / \mathrm{cm}^{3}$, with $3.0 \mathrm{MA}$ on the $1.0 \mathrm{~cm}$-radius copper inner conductor, with the outer aluminum liner starting at $\sim 5.4 \mathrm{~cm}$ inner radius $(0.6 \mathrm{~cm}$ thick). Current driving this liner was based on a portion of the measured HEL-1 current, which would implode this liner from 5.4 $\mathrm{cm}$ to $1.36 \mathrm{~cm}$ in $7.5 \mu \mathrm{sec}$, with a final implosion velocity of $1.6 \mathrm{~cm} / \mu \mathrm{sec}$ (computed liner inner radius vs. time values were used in the MHRDR calculation). Liner-on-plasma implosions using liners driven by the Atlas facility have also been calculated. Such liner implosions are not necessarily optimized choices for an experiment intended to achieve fusion conditions, but they represent clearly achievable goals which could serve as useful steps in demonstrating compression heating of an MTF plasma.

For an equivalent volumetric adiabatic compression (cylindrical), the temperature of the gas would go from $100 \mathrm{eV}$ to $1.03 \mathrm{keV}$. A MHRDR one-dimensional, twotemperature calculation, which includes radiative and conductive heat losses, reaches a mass-weighted average temperature $\left(\left\langle T_{i}>\sim<T_{e}>\right)\right.$ of $620 \mathrm{eV}$, with a final radial temperature profile peaking at $900 \mathrm{eV}$. A MHRDR 
three-temperature calculation, starting with a radiation temperature of $1 \mathrm{eV}$, with open boundaries, duplicates this result, confirming our usual assertion that the DT plasma is optically thin, so that two-temperature calculations with a radiative energy loss term are reasonable. A two-temperature MHRDR calculation, with Braginskii electron heat conduction and nonmagnetized ion heat conduction multiplied times an arbitrary factor of $1 / 15$, also gave a final peak temperature of $900 \mathrm{eV}$; this multiplier was then used in calculations with the other Los Alamos code to approximate the Braginskii ion insulation effect.

One-dimensional calculations with the second MHD code show similar final plasma conditions in the target plasma, although the peak temperature reached is slightly under $800 \mathrm{eV}$, compared to the idealized single-material MHRDR calculation's peak of $900 \mathrm{eV}$. The multi-material calculation does show substantial diffusion of heat and field into the inner and outer metal walls, leading to wall temperatures as high as $40 \mathrm{eV}$. Plasma pressure is high enough that the inner rod has been compressed from its initial radius of $1.0 \mathrm{~cm}$ to about $0.93 \mathrm{~cm}$.

MHRDR has also been used to compute one- and two-dimensional compressions of computed late-time MAGO target plasmas at $2 \mathrm{~cm} / \mu \mathrm{sec}$. The starting conditions for these calculations were computed secondchamber plasmas at $12 \mu \mathrm{sec}$ in the LANL-VNIIEF MAGO-2 target plasma experiment; the MAGO-2 target plasma calculations showed good agreement to the earlier time experimental measurements available [7-11]. The computed late-time MAGO plasma profiles are diffuse, wall-supported Z-pinch equilibria which show Kadomtsev stability to $m=0$ perturbations. In liner-onplasma calculations, the $10-\mathrm{cm}$ outer wall was imploded at $2 \mathrm{~cm} / \mu \mathrm{sec}$ to a final radius of $1.4 \mathrm{~cm}$ (inner wall was $1.2 \mathrm{~cm}$ ). One-dimensional calculations reached a peak mass-weighted average temperature of $4.75 \mathrm{keV}$, with a peak profile temperature of $7 \mathrm{keV}$. Two-dimensional calculations have been run as far as $3.5 \mu \mathrm{sec}$ to date, with the $\langle\mathrm{T}\rangle$ the same as in the one-dimensional result $(800$ $\mathrm{eV})$. An interesting feature can be seen in the twodimensional calculation: formation of convective cooling cells close to the imploding outer boundary. Since the bulk temperature reached has not changed compared to the one-dimensional case, this appears (to the time calculated to date) to be a localized effect countered by stronger heating and insulating processes. Increasingly detailed two-dimensional multi-material simulations of MAGO plasmas imploded by Atlas or HEL-1-like liners are also in progress with the second Los Alamos MHD code. These calculations promise to shed some light on wall heating and other phenomena to be expected in such experiments.

Will ionized wall material mix with and cool DT plasma before it can be compressively heated to fusion conditions and produce significant fusion energy? The answer to this question depends upon the rates of the competing processes of implosion, heating, mixing, and cooling. Detailed calculations, which must ultimately be validated by experiment, can answer this question. The codes described here contain substantial portions of the physics governing these competing processes, including potential two-dimensional effects. Guided by experimental data as it becomes available, we can utilize these tools to predict the important issues for optimizing the design of future MTF liner-on-plasma demonstration experiments.

\section{CONCLUSIONS}

Magnetized Target Fusion (MTF) is an approach to controlled fusion which potentially avoids the difficulties of the traditional magnetic and inertial confinement approaches. It appears possible to investigate the critical issues for MTF at low cost, relative to traditional fusion programs, utilizing pulsed power drivers much less expensive than ICF drivers, and plasma configurations much less expensive than those needed for full magnetic confinement. Computational modeling of separate MTF target plasma and liner implosion experiments has shown good agreement to experiment. Combining target plasma and liner implosion computational models allows detailed theoretical investigation of important issues for proposed MTF liner-on-plasma experiments.

\section{REFERENCES}

[1] I. Lindemuth and R. Kirkpatrick, Nuclear Fusion 23, 263 (1983).

[2] V. Mokhov et al., Sov. Phys. Dokl. 24, 557 (1979).

[3] V. Chernyshev et al., in Megagauss Fields and Pulsed Power Systems, ed. V. Titov \& G. Shvetsov, p. 347, Nova Science Publishers, NY(1990).

[4] A. Pavlovskii et al., ibid., p. 327.

[5] V. Chernyshev et al., in Megagauss Magnetic Field Generation and Pulsed Power Applications, ed. M. Cowan and R. Spielman, Part 1, p. 557, Nova Science Publishers, New York (1995).

[6] I. Lindemuth et al., ibid., p. 361.

[7] P. Sheehey et al., in Megagauss-VII Proceedings, Saroy, Russia (1996).

[8] R. Reinovsky et al., ibid.

[9] I. Lindemuth, V. Chernyshev, V. Mokhov et al., Physical Review Letters 75(10), 1953 (1995).

[10] P. Sheehey et al., Fusion Technology 30, 1355 (1996).

[11] P. Sheehey et al., in Megagauss-VIII Proceedings, Tallahassee, FL (1998).

[12] R. Faehl et al., in Proceedings of the $11^{\text {th }}$ International Pulsed Power Conference, Baltimore, MD, p. 1375 (1997).

[13] P. Sheehey and I. Lindemuth, Physics of Plasmas 4, $146(1997)$.

[14] P. Sheehey et al., in Dense Z-Pinches, $4^{\text {th }}$ International Conference, AIP Conference Proceedings 409, p.17 (1997).

[15] I. Lindemuth and M. Widner, Physics of Fluids 24, 746 (1981).

[16] R. Bowers et al., J. Appl. Phys. 83, 4146 (1998).

[17] R. Milroy and J. Brackbill, Physics of Fluids 25, 775 (1982). 

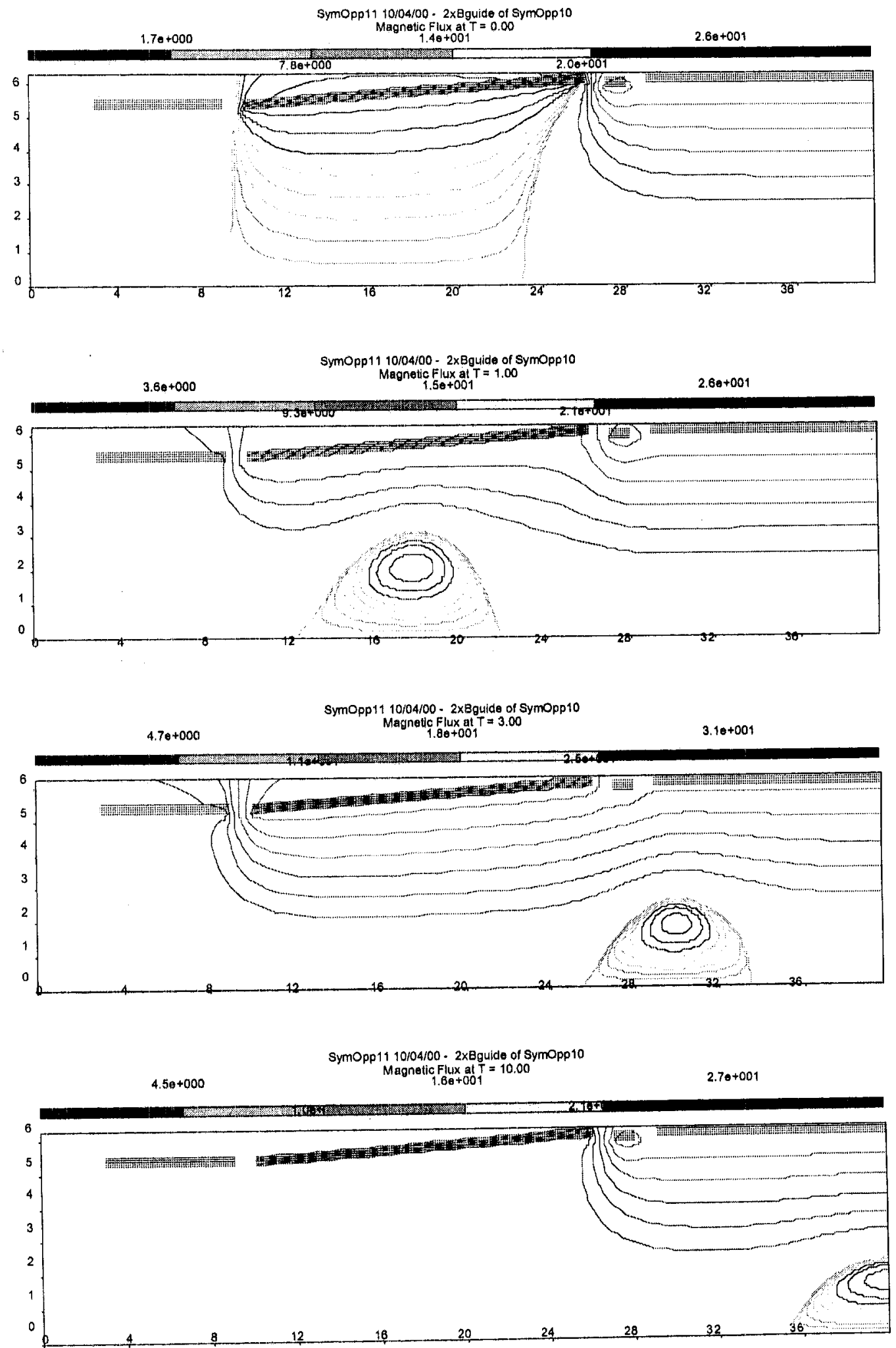

Figure 1. MOQUI calculation of FRC formation and translation into liner implosion chamber. 